nimmt, sie eine Periode lang begleitet und dann wieder an die „rechte“ Hälfte der nächsten Welle abgibt. Die Stetigkeit ist also durch die Mitwirkung der zirkulierenden Welle selbst gewahrt.

Im Zentrum $x^{\prime}=0, z^{\prime}=0$ verschwindet die zirkulierende Welle, wie aus den oben angeschriebenen Gleichungen hervorgeht. Die Energieströmung ist in der Umgebung des Zentrums sehr klein; daher wurden die Poynting-Vektoren dort 10-fach verlängert gezeichnet (angemerkt durch einen Schrägstrich durch die Pfeile), auch am Sattelpunkt verschwindet die Energieströmung; die Poynting-Vektoren wurden dort 100-fach verlängert (angemerkt durch 2 Schrägstriche durch den Pfeil). Wenn man den Phasenflächen eine Strichdicke entsprechend einem bestimmten Zeitintervall geben würde, so müßte die Umgebung des Sattelpunktes bis zu den dick asteroideartigen Kurven schwarz gezeichnet werden.

Die zirkulierende Welle ist eine in der Optik zwar neue, aber durchaus unkomplizierte Erscheinung; ist sie mathematisch doch einfach durch geeignete Addition von sechs ebenen Wellen, vier homogenen und zwei quergedämpften darzustellen. Eine sehr ähnliche Zirkulation ergibt sich sogar aus vier homogenen, ebenen Wellen allein, wie sie im dichteren Medium bei der Totalreflexion einer mit Nullebenen gekennzeichneten Welle auftreten. Berechnet und zeichnet man nämlich die hier in der Umgebung des Null- punktes dargestellten Phasenflächen auch weiter im Glasinnern für die vollständige - aus einfallender und reflektierter Welle zusammengesetzte - Erregung nach Gl. (12), so erhält man die Abb. 4. Diese zeigt außer dem in Abb. 3 Dargestellten darüber noch vier weitere der abzählbar vielen Zirkulationszentren. Die Energiestromlinien sind wieder als orthogonale Trajektoren zu denken. An jedem Zirkulationszentrum erfolgt ein Schwenken der Phasenflächen und ein Umlauf von einer Periode. Diese dauernden Schwenkungen sind offenbar erforderlich, um das Ausströmen der Energie aus dem dünneren Medium links der Achse $x^{\prime}=0$ und ein Zurückströmen rechts von ihr zu ermöglichen, ohne daß es zu Unstetigkeiten kommt. Dieses Hin und Her der Energie tritt bei der ungekennzeichneten - also unbegrenzten einfachen ebenen Welle - nicht auf, wie ja bekannt ist; dort laufen alle $\overline{\mathfrak{S}}$-Linien in beiden Medien parallel der Grenzfläche. Die Zirkulationen bei der Totalreflexion (und ähnlich in anderen Fällen, die hier nicht diskutiert werden mögen) sind ersichtlich eine notwendige Begleiterscheinung der Strahlkennzeichnung selbst. Das läßt das Auftreten solcher Zirkulationen auch bei anderen als den hier zur Strahlkennzeichnung benutzten Ungleichmäßigkeiten der total zu reflektierenden Welle vermuten.

Verf. dankt Hrn. Prof. Dr. L o c h t e - Holt greven für die liebenswürdige Unterstützung seiner Arbeit.

\title{
Über mögliche Strukturabhängigkeiten des Metallelektrodenpotentials
}

\author{
Von WOLFGang LORENZ \\ Aus dem Physikalischen Institut der Universität Leipzig \\ (Z. Naturforschg. 5 a, 283-291 [1950]; eingegangen am 4. April 1950)
}

Die Abhängigkeit des Metallelektrodenpotentials von Kristallgröße und kristallographischer Oberflächenstruktur wird theoretisch untersucht. Auf Grund der statistisch-thermodynamischen Theorie der Elektrodenpotentiale, der Kossel-Stranskischen Kristallwachstumstheorie und einiger Ergebnisse der Elektronenemissionsforschung ist folgendes zu erwarten:

1. Eine Verringerung der mittleren Atomabtrennungsarbeit, die bei sehr kleinen Kristallen auftritt, bewirkt eine Potentialverunedlung.

2. Die Anisotropie der Elektronenaustrittsarbeit führt zu Potentialunterschieden zwischen verschiedenen Kristallflächenarten. Für das Elektrodenpotential sind dabei nicht die beobachtbaren Flächenaustrittsarbeiten, sondern die Austrittsarbeiten aus den zugeordneten Wachstumsstellen maßgebend.

3. Die Gleichgewichtspotentiale sollten im allgemeinen durch Elektrolytadsorption nicht beeinflußt werden.

4. Das Gleichgewicht eines Kristalls in Elektrolytlösung wird durch die Anisotropie des Elektrodenpotentials bestimmt; danach ist nur die Flächenart mit dem negativsten Potential stabil.

5. Das absolute Potential erfährt in seinem theoretischen Wert eine Änderung.

Es werden Möglichkeiten besprochen, die die Anwendbarkeit der Theorie einschränken können, und zum Schluß verschiedene Beobachtungen im Sinne der entwickelten Theorie neu gedeutet. 
I.

$\mathrm{D}$ aß bei der elektrolytischen Metallabscheidung und -auflösung der kristalline Bau der Metalloberfläche eine wichtige Rolle spielt, ist erst verhältnismäßig spät erkannt worden, nachdem Volme ${ }^{1}$, $\mathrm{Kossel}{ }^{2}$ und $\mathrm{Stranski}{ }^{3}$ in grundlegenden Betrachtungen die Energieverhältnisse beim Kristallwachstum klargelegt hatten. Die hierdurch veranlaßte erneute Untersuchung der Elektrodenvorgänge ${ }^{4}$ zeigte nämlich, daß die bei kathodischer oder anodischer Polarisation auftretende Überspannung im allgemeinen durch verzögerten Ladungsübertritt an der Metalloberfläche und - da der Übertritt mit großer Wahrscheinlichkeit erst an den Wachstumsstellen erfolgt durch Hemmungen des Kristallwachstums bzw. -abbaus zu erklären ist ${ }^{5}$, während man vorher ${ }^{6}$ dazu neigte, die Ionendehydratation in der Lösung als geschwindigkeitsbestimmend anzusehen ${ }^{7}$. Die Transporthemmungen bei der Elektrolyse werden also durch die Oberflächenstruktur des Metalls sicher beeinflußt, wenn auch genauere Einzelheiten, z. B. die unterschiedliche Polarisation an verschiedenen kristallographischen Flächen eines Metallkristalls, noch nicht untersucht sind.

$\mathrm{Da}$ in entsprechender Weise auch die Gleichgewichtspotentiale orientierungsabhängig sein könnten, ist bisher kaum ${ }^{8}$ in Betracht gezogen worden, obwohl gerade diese Frage von grundlegender Bedeutung ist. Sie soll deshalb im folgenden zusammen mit der Frage des Einflusses der Kristallgröße eingehender besprochen werden.

\section{Strukturabhängigkeit des Elektrodenpotentials}

Um eine Abhängigkeit des Metallelektrodenpotentials von Kristallgröße und Oberflächenbau einzusehen, gehen wir in Anlehnung an die bekannte theoretische Behandlung der Elektrodenpotentiale durch $\mathrm{Makishima}{ }^{9}$ so vor, daß wir einen reversiblen Kreisprozeß betrachten, in dem das Elektrodenmetall

1 M. Volm e r, Z. physik. Chem. 102, 267 [1922].

2 W. Koss el, Gött. Nachr. 1927, 135; Quantentheorie und Chemie (Leipziger Vorträge 1928, herausgeg. von H. Falkenhagen, S. 1).

3 I. N. S tr a n s k i, Z. physik. Chemie 136, 259 [1928].

4 H. B randes, Z. physik. Chem. Abt. A 142, 97 [1929]; T. Erde y-Gruz u. M. Volmer, Z. physik. Chem., Abt. A 157, 165 [1931]; M. Volmer, Physik. Z. Sowjetunion 4, 346 [1933]; Das elektrolytische Kristallwachstum, Hermann u. Cie., Paris 1934. Ferner W. A. Rojter, W. A. Juza u. E.S. Poluj a n, Acta physicochim. URSS 10, 389, 845 [1939]; Chem. Zbl. 1943, II, 804. verdampft, der Dampf ionisiert, in Lösung überführt und schließlich das solvatisierte Ion durch den Elektrodenprozeß in den Ausgangszustand zurückgebracht wird. Werden dabei die drei ersten Übergänge so geleitet, daß keine Änderung der freien Energie auftritt, so ist die Änderung der freien Energie beim letzten, dem Elektrodenprozeß ebenfalls null, d. h. die Potentialdifferenz zwischen Metall und Elektrolyt

$$
E \equiv E^{0}+\frac{R T}{n F} \ln a=0
$$

( $E^{0}$ absolutes Normalpotential, bezogen auf Aktivität $a=1$ der Ionen in Lösung). Aus der Aktivität $a$ im 3. Zwischenzustand erhält man also sofort $E^{0}$. Indem wir bezüglich der genauen Durchführung der Berechnung von $a$ auf $\mathrm{M} \mathrm{a} \mathrm{kis} \mathrm{hi} \mathrm{m} \mathrm{a}{ }^{9}$ verweisen, notieren wir hier nur die Endformel für das absolute Normalpotential ${ }^{10}$; sie lautet abgekürzt

$$
E^{0}=\frac{L}{n F}-\psi+\frac{A}{n F}-\frac{S}{n F}-R,
$$

wo $L$ die molare Verdampfungswärme, $\psi$ die Elektronenaustrittsarbeit $[\mathrm{eV}], A$ die Ionisierungsarbeit des $n$-wertigen Ions, $S$ die Solvatationswärme bedeutet; in $R$ sind einige für das folgende weniger wichtige Terme zusammengefaßt (chem. Konstante u. a.).

Im folgenden beschränken wir uns darauf, den Kreisprozeß in Hinsicht auf die erwähnten Strukturabhängigkeiten zu untersuchen. Dabei müssen wir darauf achten, daß wir die am Kristall stattfindenden Übergänge der ersten drei Teilprozesse an den gleichen Stellen der Kristalloberfläche vornehmen, die beim letzten Teilprozeß, dem uns interessierenden Elektrodenprozeß, in umgekehrter Richtung durchschritten werden. Da wir dabei verschiedene Vorstellungen der Kristalloberflächenkinetik heranziehen

5 Bei der Wasserstoffabscheidung erfolgt nach der Icnenentladung noch die $\mathrm{H}_{2}$-Molekülbildung; dieser Teilvorgang scheint gegenüber der Entladung wenig Einfluß zu haben, s. K. Wirt z, Z. Elektrochem. angew. physik. Chem. 44, 303 [1938].

6 M. Le Blanc, Abh. Bunsen-Ges. 3 [1910].

7 Vgl. hierzu G. M a sing, Z. Elektrochem. angew. physik. Chem. 48, 85 [1942].

8 s. u. Anm. 54-56. Im Zusammenhang mit Elektronenemissionsfragen wies ferner R. S u h r m a n n gelegentlich auf eine solche Möglichkeit hin, s. Z. Elektrochem. angew. physik. Chem. 44, 478 [1938].

9 S. M a k is hi m a, Z. Elektrochem. angew. physik. Chem. 41, 697 [1935]. Ähnliche Betrachtungen bereits bei K. F a j a n s, Verh. dtsch. physik. Ges. 1919, 549, 709; und E. L a n g e u. K. P. M i s c e n k o, Z. physik. Chem. Abt. A 149, 1 [1930].

10 Vgl. auch A. E u c k e n, Lehrb. d. Chem. Physik II, 2 [1944], S. 1322. 
müssen, wollen wir das für uns Nötige hierüber kurz vorausschicken.

Die Tatsache, daß unter den wiederholbaren Anlagerungsmöglichkeiten an eine Kristallfläche die Anlagerung an den „Halbkristall“" 11 die energiereichste ist, hat zur Folge, daß diese Stelle bevorzugt als Wachstumsstelle fungiert; durch Oberflächendiffusion ${ }^{12}$ werden woanders auftreffende, schwächer gebundene Teilchen verhältnismäßig leicht in diese Lage befördert. Bei nichtpolaren Kristallen ${ }^{11,13}$, mit denen wir es ausschließlich zu tun haben werden, macht man dabei zur Abschätzung der Bindungsverhältnisse die vereinfachende Annahme, daß die Abtrennungsarbeit eines Oberflächenatoms der Zahl seiner Nachbarn proportional ist: man schreibt die Nachbarzahlen nach zunehmendem Abstand im Schema $n_{1}\left|n_{2}\right| n_{3} \mid \ldots$ auf (Kossel) und ordnet den Spalten abnehmende Energiebeträge $\varphi_{i} \mathrm{zu}$, welche der Abtrennung von einem Nachbarn in der jeweiligen Entfernung entsprechen ${ }^{14}$. Dieser additive Ansatz wird durch den nebenvalenzartigen, nichtabsättigbaren Charakter der metallischen Bindung ${ }^{15}$ weitgehend gerechtfertigt, wenn auch nicht exakt, wie die durch röntgenographische Fourieranalyse ermittelte Elektronendichteverteilung zeigt; doch liegen die Verhältnisse in der Metalloberfläche möglicherweise günstiger als im Metallinnern, so daß gelegentlich geäußerte Bedenken ${ }^{16}$ nicht allzu schwerwiegend sind, zumal zwischen der theoretisch geforderten und der experimentell beobachteten Kristallgleichgewichtsform bei einfachen Gittern völlige, bei weniger symmetrischen Gittern weitgehende Übereinstimmung besteht ${ }^{17}$. In der Theorie metallischer Mischphasen (Bragg-Williams, Bethe u. a.) benutzt man übrigens analoge Ansätze ebenfalls mit Erfolg.

11 W. K o s s e 1 1, Leipz. Vortr. 1928, 1.

12 M. V o l m e r u. G. A d hikari, Z. physik. Chem. 119, 46 [1926]; Z. Physik 35, 170 [1926]; K. N e u m a n n, Z. Elektrochem. angew. physik. Chem. 44, 474 [1938]; M. Volmer, Kinetik der Phasenbildung. Steinkopff, Dresden 1939.

13 I. N. S trans ki, Z. physik. Chem. Abt. B 11, 342 [1930]; Physik. Z. 36, 393 [1935] (dort weitere Literatur); Ann. Physik 23, 330 [1935].

14 Das Verhältnis $\varphi_{2} / \varphi_{1}$ ist umso kleiner, je größer das Entfernungsverhältnis $r_{2} / r_{1}$ der Nachbarn ist. Bei kub. rz. Gittern ist $r_{2} / r_{1}=1,15$, bei kub. flz. und hex. dicht. Pakkungen $=1,41$. I. N. Stranski, Z. physik. Chem. Abt. B 38, 451 [1938], bestimmte $\varphi_{2} / \varphi_{1}$ aus dem Verschwinden von Flächen an der Gleichgewichtsform von Cd-Kristallen (hex. dicht.) bei zunehmender Metalldampfübersättigung $\mathrm{zu} \sim 0,04$, während bei kub. rz. Gittern $\varphi_{2} / \varphi_{1}$ von der Größenordnung 0,5 sein dürfte, s. I. N. S t r a n s ki u. R. S u hrmann, Ann. Physik 1, 153 [1947]. $\varphi_{3} / \varphi_{1}$ ist noch wenig $>0, \varphi_{4} / \varphi_{1}$ allgemein $=0$.

15 U. D e hling er, Z. Elektrochem. angew. physik. Chem. 46, 402 [1940].
Nach dieser Zwischenbemerkung wenden wir uns nun dem oben beschriebenen Kreisprozeß zu und betrachten der Reihe nach die in ihm auftretenden Teilübergänge.

\section{a) Isotherme Verdampfung des Metalls unter seinem eigenen Dampfdruck}

Setzen wir einen Kristall voraus, der von hinreichend großen glatten Flächen begrenzt ist, so ist die Abtrennungsarbeit eines Atoms an jeder Fläche dieselbe, nämlich gleich der Abtrennungsarbeit aus der Halbkristallage $\varphi_{1 / 2}$. Nur bei sehr kleinen Kristallflächen weicht die mittlere Abtrennungsarbeit $\bar{\varphi}$ infolge der an den Flächenrändern geringeren Abtrennungsarbeit merklich von $\varphi_{1 / 2} \mathrm{ab}$, was zu einer Dampfdruckerhöhung gemäß der Thomson-Gibbsschen Gleichung ${ }^{18}$ führt. Bei Kristallen von einer Kantenlänge von $10^{2}$ Atomen entspricht dieser Erhöhung eine Potentialverschiebung ${ }^{19}$ um etwa $10^{-2}$ bis $10^{-1} \mathrm{~V}$ nach der negativen Seite. Pótentialdifferenzen zwischen verschiedenen Flächen eines in der Nähe des thermodynamischen Gleichgewichts befindlichen Kristalls entstehen hierdurch praktisch nicht, da das Gleichgewicht durch die Gleichheit der $\bar{\varphi}$ an allen Flächen definiert ist. Irreversible Gitterstörungen lassen wir außer Betracht, sie sind bei Kristallen, die in größerem Umfang einem Wachstum bzw. einer Auflösung unterworfen werden, sicher auch nicht wesentlich.

\section{b) Ionisierung der Dampfatome}

im Gleichgewicht mit dem Metallelektronengas

Das Ionisierungsgleichgewicht wird durch die Metalldampfdichte und die Elektronendichte im Dampfraum bestimmt; die Dichte der Elektronen im Gleichgewicht mit dem Elektronengas im Metall hängt dabei wesentlich von der Elektronenaustrittsarbeit $a b$, die in das Normalpotential linear eingeht. Nun ist bekannt, daß die Elektronenemission nicht nur durch adsorbierte Fremdatome, sondern auch durch die kristallographische Struktur der Oberfläche stark beeinflußt wird; wir müssen uns deshalb mit dieser Erscheinung ausführlich beschäftigen, da sie zu

16 A. E u cke n, Lehrb. d. Chem. Physik II, 2 [1944], S. 1180, 1183, Anm. 1.

17 Vgl. die zusammenfassende Übersicht von M. S tra umanis, in G.-M. S chwab, Handbuch der Katalyse Bd. 4, Springer-Verlag, Wien 1943, S. 269.

18 I. N. Stranski u. R. Ka i s chew, Physik. Z. 36, 393 [1935].

19 Nach (2), wobei man als Verdampfungswärme $L=N_{\mathrm{L}} \bar{\varphi}$ $+R T$ und als Verringerung der Verdampfungswärme $\Delta L=N_{\mathrm{L}}\left(\varphi_{1 / 2}-\bar{\varphi}\right)$ hat. 


\begin{tabular}{|c|c|c|c|c|c|}
\hline Metall & Gittertyp & Fläche & $\begin{array}{l}\text { Austritts- } \\
\text { arbeit }[\mathrm{eV}]\end{array}$ & Autor & Meßverfahren \\
\hline $\mathrm{Zn}$ & hex.d.P. & $\begin{array}{l}(0001) \\
(10 \overline{1} 0)\end{array}$ & $\begin{array}{l}3,28 \\
3,09\end{array}$ & $\begin{array}{l}\text { Nitzsche }^{23} \\
\text { Nitzsche }^{23}\end{array}$ & $\begin{array}{l}\text { Photoeffekt } \\
\text { Photoeffekt }\end{array}$ \\
\hline $\mathrm{Cu}$ & kub. flz. & $\begin{array}{l}(111) \\
(001)\end{array}$ & $\begin{array}{l}4,86 \\
5,62\end{array}$ & $\begin{array}{l}\text { Underwood }^{24} \\
\text { Underwood }^{24}\end{array}$ & $\begin{array}{l}\text { Photoeffekt } \\
\text { Photceffekt }\end{array}$ \\
\hline $\mathrm{Ag}$ & kub. flz. & $\begin{array}{l}(111) \\
(001)\end{array}$ & $\begin{array}{l}4,75 \\
4,81\end{array}$ & $\begin{array}{l}\text { Farnsworth } \\
\text { Farnsworth }\end{array}$ & $\begin{array}{l}\text { Photoeffekt } \\
\text { Photoeffekt }\end{array}$ \\
\hline W & kub.rz. & $\begin{array}{l}(011) \\
(011) \\
(112) \\
(112) \\
(001) \\
(111) \\
(116)\end{array}$ & $\begin{array}{l}6,0 \\
(4,65)^{29} \\
4,66 \\
(4,50)^{30} \\
4,53 \\
4,36 \\
4,36\end{array}$ & $\begin{array}{l}\text { Müller }{ }^{26} \\
\text { Nichols }^{27} \\
\text { Nichols }^{27} \\
\text { Mendenhall }^{28} \\
\text { Nichols }^{27} \\
\text { Nichols }^{27} \\
\text { Nichols }^{27}\end{array}$ & $\begin{array}{l}\text { Feldemission } \\
\text { Glühemission } \\
\text { Glühemission } \\
\text { Photoeffekt } \\
\text { Glühemission } \\
\text { Glühemission } \\
\text { Glühemission }\end{array}$ \\
\hline
\end{tabular}

Tab. 1.

Effekten führt, die in der Größenordnung von $\sim 0,1 \mathrm{~V}$ liegen.

Nach der heutigen, zuerst von Wig ne r und B a r d e e ${ }^{20}$ entwickelten Auffassung setzt sich die Elektronenaustrittsarbeit zusammen aus der Abtrennarbeit aus dem Metallgitterverband und der Durchtrittsarbeit durch eine Oberflächendoppelschicht. Dabei ist nur der erste Anteil unabhängig von der Oberflächenstruktur, während der zweite an verschiedenen Kristallflächen verschieden sein kann21. Allerdings ist es bisher nicht gelungen, die Doppelschichten an verschiedenen Wolfram-Einkristallfä̈chen in genügender Übereinstimmung mit Meßwerten zu berechnen, was jedoch nur an nicht ganz zutreffenden speziellen Ansätzen liegt. Es scheint, daß eine befriedigende Deutung der Oberflächendoppelschicht und damit der gesamten Elektronenaustrittsarbeit erst durch Berücksichtigung der Tammschen Oberflächenterme möglich wird. Auf jeden Fall aber, und das ist für das hier zu besprechende Problem wesentlich, ist auf Grund dieser Theorie verschiedenen Kristallflächenarten ein verschiedener Elektronendampfdruck zugeordnet. Andere Deutungen der Richtungsabhängigkeit der Elektronenemission, die diese als rein kinetische Erscheinung betrachten und an einer Konstanz des Elektronendampfdrucks festhalten ${ }^{22}$, haben sich als unzulänglich erwiesen 21 .

In Tab. 1 sind die an definierten Kristallflächen gemessenen Austrittsarbeiten der bisher untersuchten

20 E. Wi g n e r u. J. B a r d e e n, Physic. Rev. 48, 84 [1935]; J. B a r d e e n, Physic. Rev. 49, 653 [1936].

21 R. S m o l u c h ow s k i, Physic. Rev. 60, 661 [1941]. 22 B. M r ow k a, Z. techn. Physik 18, 572 [1937]; vgl. Diskussionsbemerkung W. S c h o t k y, Z. techn. Physik 19, 19 [1938] (Orientierungsabhängige Elektronenreflexion an Oberfläche); E. W. Mülle r, Naturwiss. 27, 820 [1939] (Braggsche Reflexionen im Metallgitter).

23 A. N itz s c h e, Ann. Physik 14, 463 [1932].

24 N. Underwood, Physic. Rev. 47, 502 [1935].

25 H. E. F a rns worth u. R. P. Winch, Physic. Rev. 56, 1067 [1939]; 58, 812 [1940].
Metalle zusammengestellt; man kann daraus die Differenzen in der Austrittsarbeit verschiedener Flächen ersehen. Die aus der langwelligen Grenze des Photoeffektes bestimmten Werte sind hierbei am wenigsten sicher, da, besonders bei dem niedrig schmelzenden $\mathrm{Zn}$, die Entgasung der Oberfläche schwierig ist. An Hand der im Augenblick wohl genauesten Messungen von $\mathrm{Nichols}{ }^{27}$ an W stellten kürzlich $\mathrm{Stranski}$ u. Suhrmann ${ }^{31}$ heuristische Betrachtungen über mögliche Zusammenhänge zwischen Austrittsarbeit und anderen Kristalloberflächengrößen an, wobei sich ein entgegengesetzter Verlauf von spezifischer Oberflächenenergie und Austrittsarbeit als brauchbarster Anhaltspunkt erwies. Derselbe Gang ist auch beim hexagonalen $\mathrm{Zn}$ vorhanden, bei dem die Basisfläche (0001) die geringste Oberflächenenergie und größte Austrittsarbeit hat. Bei den kubisch flächenzentrierten Metallen $\mathrm{Cu}$ und $\mathrm{Ag}$ hingegen hat die Fläche geringster Oberflächenenergie (111) eine kleinere Austrittsarbeit als die Würfelfläche (001), eine recht überraschende Tatsache, die sicher reell ist, da die Messungen nach außerordentlich sorgfältiger Entgasung erfolgten und auch frühere

26 E. W. M ü ll e r, Z. Physik 120, 261 [1943].

27 M. H. N i chols, Physic. Rev. 57, 297 [1940].

28 C. E. Mendenhall u. C. F. de Voe, Physic. Rev. 51, 346 [1937].

29 Dieser Wert ist sicherlich nicht der Fläche (011) zuzuordnen, sondern Stufen (112) in dieser Fläche, wie Nichols selbst bemerkt. Vgl. I. N. Stranski u. R. Suhrmann, Ann. Physik 1, 153 [1947].

30 Anfangswert, Austrittsarbeit mit der Zeit beträchtlich ansteigend.

31 I. N. Stranski u. R. S uhrmann, Ann. Physik 1, 153 [1947] 
Beobachtungen bei $\mathrm{Cu}^{32}$ in dieselbe Richtung weisen. Wir wollen deshalb für das Folgende die StranskiSuhrmannsche Hypothese auf kubisch raumzentrierte Gitter und hexagonale dichteste Packungen einschränken und bei kubisch flächenzentrierten Gittern annehmen, daß (001) die größte Austrittsarbeit hat; die Ätzfiguren an dem ebenfalls kubisch flächenzentrierten $\mathrm{Al}$ lassen sich dann leicht erklären, wenn man bei Al dieselbe Anomalie in der Austrittsarbeit wie bei $\mathrm{Ag}$ und $\mathrm{Cu}$ annimmt; wir kommen darauf noch zurück.

Zunächst aber haben wir zu untersuchen, wie sich die übrigens bei zahlreichen weiteren Metallen qualitativ festgestellte Flächenabhängigkeit der Elektronenemission $^{33}$ auf das Elektrodenpotential auswirkt. In Gl. (2) ist die Elektronenaustrittsarbeit $\psi$ linear enthalten, und zwar entspricht einer Erhöhung von $\psi$ eine Potentialverschiebung nach der negativen Seite. Setzt man jetzt an Stelle der bisher benutzten Austrittsarbeit am polykristallinen Metall die an den einzelnen Flächen gemessenen Austrittsarbeiten an, so verschieben sich die absoluten Potentialwerte und es ergeben sich zwischen den verschiedenen Kristallflächenarten sehr beträchtliche Potentialdifferenzen, z. B. bei Cu zwischen (001) und (111) 0,8 V.

Dieser Ansatz ist jedoch noch nicht streng richtig. Wir müssen ja die Elektronenemission, die vom Austrittsort abhängt, an der gle:chen Stelle vornehmen, an der die Ionenentladung erfolgt. Nach $\mathrm{V}$ ol m e r ${ }^{34}$ findet nun die Entladung höchstwahrscheinlich erst an den Wachstumsstellen des Kristalles statt: Der Ionencharakter bleibt bei der Diffusion an der Metalloberfläche bis zum endgültigen Einbau ins Gitter erhalten, wie aus der Unabhängigkeit der Polarisationskapazität von einer Vorpolarisation geschlossen wird. Danach müssen wir also die Elektronenemission aus der an einer bestimmten Fläche befindlichen $\mathrm{Halb}$ kristall-Lage als maßgebend für den Elektrodenprozeß ansehen.

Über die Austrittsarbeit aus den Halbkristall-Lagen lassen sich vorläufig nur Vermutungen aussprechen. Die bisherigen Ansichten über die Emission aus Ecken und Kanten divergieren stark; teils wird eine Erhöhung von $\psi$ angenommen ${ }^{35}$, wobei man sich auf ein durch Oberflächenaufrauhung erklärtes Anwach-

32 B. A. R o s e, Physic. Rev. 44, 585 [1933]; H. E. F a r n s w o r th, Proc. Nat. Acad. Sci. 19, 777 [1933].

$33 \mathrm{Vgl}$. die elektronenmikroskopische Literatur.

34 M. Volmer, Das elektrolytische Kristallwachstum, Herman u. Cie., Paris 1934, S. $6 \mathrm{ff}$.

35 P. A. T hi e s s e n, bei R. H a u l, Z. physik. Chem. Abt. B 53, 343 [1943], Anm. 34.

36 I. N. Stranski u. R. Suhrmann ${ }^{11}$, S. 167; E. W. M ülle r, Z. Physik 108, 675 [1938]. sen von $\psi$ bei Metallschmelzen beruft, teils nimmt man, wenigstens an den Flächen hoher Austrittsarbeit, eine Erniedrigung von $\psi$ an ${ }^{36}$, gestützt auf die beobachtete Emissionszunahme bei schneller Kondensation von Wolframdampf auf W-(110)-Flächen, die mit einer Erhöhung der Ecken- und Kantenenergie verbunden ist. Die letztere Auffassung scheint allerdings besser belegt zu sein, und sie wird auch durch die eben besprochene Entladungsverzögerung nahegelegt. Solange es aber nicht gelungen ist, die Strukturabhängigkeit der Elektronenemission in befriedigender Weise quantenmechanisch zu behandeln, dürften genauere Aussagen speziell über die Austrittsarbeiten an den verschiedenen Halbkristalllagen ${ }^{37}$ nicht möglich sein. Wir fassen deshalb unsere Betrachtungen dahingehend zusammen, daß infolge der Abhängigkeit der Elektronenemission vom Kristalloberflächenbau den verschiedenen Flächenarten verschiedene Gleichgewichtspotentiale zukommen müssen. Für das Elektrodenpotential maßgebend ist dabei die Austrittsarbeit aus der Halbkristall-Lage einer Fläche, die wahrscheinlich kleiner ist als die Austrittsarbeit aus der Fläche selbst; die Reihenfolge der Austrittsarbeiten aus den Wachstumsstellen dürfte der Reihenfolge der entsprechenden Flächenaustrittsarbeiten ziemlich parallel gehen. Die auftretenden Potentialdifferenzen dürften sich etwa in der Größenordnung $10^{-1} \mathrm{~V}$ bewegen.

\section{c) Überführung der Metallionen in Lösung}

Bei diesem 3. Teilprozeß ist die Kristalloberfläche nicht beteiligt und daher kein Einfluß der Oberflächenstruktur vorhanden. Bemerkt werden muß lediglich, daß der Übergang der Ionen aus einer lösungsmitteldampffreien Atmosphäre erfolgt, wodurch der Zweifel behoben wird, ob man im 2. Teilprozeß die Austrittsarbeit ins metalldampfgesättigte Vakuum benutzen darf.

Damit ist die uns interessierende erste Hälfte des Kreisprozesses beendet; die Anisotropie der Elektronenaustrittsarbeit erweist sich dabei als Grund für eine Flächenabhängigkeit des Elektrodenpotentials. Bevor wir nun dazu übergehen, aus den gewonnenen Ergebnissen einige Folgerungen zu ziehen, müssen

37 Während Abtrennungsarbeit und Nachbarnzahl an den Halbkristall-Lagen sämtlicher Flächen übereinstimmen, sind die entsprechenden Elektronenaustrittsarbeiten sicher nicht gleich, da die räumliche Anordnung im allg. bereits der erstnächsten Nachbarn in den einzelnen Halbkristall-Lagen Unterschiede aufweist, die sich in $\psi$ auswirken müssen. 
wir über die bisher nicht beachtete Adsorption von Elektrolytbestandteilen an der Metalloberfläche noch einige Worte sagen.

\section{Einflußder Adsorption}

Wir bemerken gleich vorweg, daß die Gleichgewichtspotentiale durch Elektrolytadsorption an der Metalloberfläche in weitgehender Näherung unbeeinflußt bleiben müssen. Das läßt sich leicht begründen: Wir erweitern den Kreisprozeß dadurch, daß wir zunächst eine Berührung von Metall und Elektrolyt annehmen. Der erste Teilprozeß ist dann die Trennung von Metall und Elektrolyt, wobei die Benetzungswärme $B$ aufzubringen ist. Hiernach folgen die drei bereits besprochenen Teilprozesse, an deren Ende $1 \mathrm{Mol}$ Metall in den vom Metall getrennten Elektrolyt übergegangen ist. Jetzt wird die Berührung zwischen Metall und Elektrolyt wiederhergestellt, wobei die vorhin aufgebrachte Benetzungsenergie $B$ zurückgewonnen wird. Schließlich wird durch den Elektrodenprozeß der Anfangszustand wieder hergestellt.

Bei Vorliegen einer Adsorption hat man nun einfach $B+A$ statt $B$ zu setzen, wo $A$ die Adsorptionsenergie ist; $A$ fällt ebenso wie $B$ aus der Energiebilanz wieder heraus.

Die Unabhängigkeit des Potentials von einer Adsorption ist auch dadurch verständlich, daß vor der Entladung eines Ions die an der Wachstumsstelle adsorbierten Teilchen desorbiert werden müssen, um dem Ion Platz zu machen. Die räumlichen Verhältnisse beim Elektronenübertritt entsprechen dann genau denen bei fehlender Adsorption, wenn man die von weiter entfernt adsorbierten Partikeln herrührenden Gitterdeformationen vernachlässigt; diese durch die hier bemutzte Methode nicht erfaßbaren Veränderungen sind aber infolge der kurzen Reichweite der Gitterkräfte sicherlich unbedeutend. Nach erfolgter Ionenentladung kann dann die Adsorption an der nunmehr um 1 Atomabstand gewanderten Wachstumsstelle neu erfolgen.

Vorausgesetzt haben wir in beiden Gedankengängen, daß die Ionenentladung nur unmittelbar am Gitter und nicht etwa durch eine eventuelle Adsorptionsschicht hindurch über mehrere Atomabstände erfolgt. Wäre das der Fall, so würde die Elektronenaustrittsarbeit und damit das Potential durch selektive Adsorption gewisser Elektrolytbestandteile natürlich in weiten Grenzen zu verändern sein. $\mathrm{Da} ß$ eine solche

38 Diese Beeinflußbarkeit müßte auch beim relativen Normalpotential vorhanden sein, s. Kap. V.

39 Vgl. C. Wa gner, in G. M a s ing, Handb. d. Metallphysik, Acad. Verlagsges., Leipzig 1940, I 2, S. 171.
Beeinflußbarkeit der Potentiale aber tatsächlich wohl kaum vorhanden ist ${ }^{38}$, läßt eine „Entladung über weite Entfernung" sehr unwahrscheinlich erscheinen. Eine Ausnahme könnte vielleicht bei der chemischen Passivität vorliegen, deren Mechanismus noch völlig ungeklärt ist ${ }^{39}$.

Die nach unserer Ansicht zur Entladung nötige Desorption des blockierenden Teilchens von der Wachstumsstelle erfordert lediglich eine u. U. beträchtliche Aktivierungsenergie, die sich in einer erhöhten kathodischen oder anodischen Überspannung auswirkt ${ }^{40}$. Die adsorptive Bindung der Elektrolytbestandteile an den verschiedenen Kristallflächenarten wird dabei aus strukturgeometrischen Gründen ${ }^{41}$ unterschiedlich sein, so daß hieraus sofort eine Flächenabhängigkeit der elektrolytischen Polarisation folgt. Dabei ist noch die Frage offen, ob die oftmals hintereinander aufzubringende Aktivierungsenergie bei der Oberflächendiffusion oder die einmalige Aktivierung zur Freilegung der Wachstumsstelle den Hauptanteil der Überspannung verursacht. Wir wollen jetzt aber hierauf nicht weiter eingehen, sondern uns den Fragen zuwenden, die sich aus den bisherigen Feststellungen ergeben.

\section{Gleichgewicht eines Kristalls in Elektrolytlösung}

Die Gleichgewichtsform eines Kristalls in reiner gesättigter Metalldampfumgebung ist dadurch bestimmt ${ }^{18}$, daß die Abtrennungsarbeit jedes Oberflächenbausteins mindestens so groß sein muß wie die Abtrennungsarbeit in den Halbkristall-Lagen. Gewisse Ecken und Kanten an einem durch die Flächen kleinster Oberflächenenergie gebildeten Polyeder sind hiernach nicht stabil und werden deshalb abgestumpft; die Ausdehnung der neu erscheinenden Flächen wird durch die erforderliche Gleichheit der mittleren $\mathrm{Ab}$ trennungsarbeit an allen Flächen festgelegt. An Metallkristallen in Dampfumgebung treten infolgedessen durchweg mehrere Flächenarten auf, und die Übereinstimmung zwischen Theorie und Experiment ist in diesem Punkt so gut als man nur wünschen kann ${ }^{17}$.

Man hat versucht, auch die Gleichgewichtsform elektrolytisch gewachsener Kristalle unter diesem Ge-

$40 \mathrm{Da}$ prinzipiell sämtliche Elektrolytbestandteile als Inhibitoren wirken können, zeigte $\mathrm{H}$. F is c h e r, Z. Elektrochem. angew. physik. Chem. 49, 343, 376 [1943]; 50, 11 [1944]; Kolloid-Z. 106, 50 [1944]; Korros. u. Metallschutz 20, 285 [1944].

41 I. N. S tra n s ki, Z. anorg. Chem. 252, 241 [1944]; I. N. Stranski u. R. S uhrmann, Ann. Physik 1, 169 [1947]. 
sichtspunkt zu behandeln ${ }^{42}$. Das ist jedoch nicht zulässig, wenn für die Anlagerung geladener Teilchen nicht nur, wie oben, die Gitterkräfte und zusätzlich die von $\mathrm{Stranski}{ }^{43}$ berücksichtigten elektrostatischen Kräfte, sondern außerdem noch die Elektronenaustrittsarbeiten als strukturabhängige Größen maßgebend sind. An der Gleichgewichtsform eines Kristalls in Elektrolytlösung sollte deshalb in makroskopischer Ausdehnung nur eine einzige Flächenart, nämlich die mit dem negativsten Potential, erscheinen ${ }^{44}$. Die Ecken und Kanten an dieser einfachen Gleichgewichtsform sind höchstens so weit abgestumpft, daß die Potentialverunedlung infolge der größeren Atomabtrennungswahrscheinlichkeit, die mit zunehmender Abstumpfung schnell abnimmt, durch die Potentialveredlung infolge der verringerten Austrittsarbeit gerade aufgewogen wird.

Bringt man eine Metalloberfläche, die infolge mechanischer oder thermischer Vorbehandlung verschiedene kristallographische Flächen enthält, mit der entsprechenden Metallionenlösung in Berührung, so sollte eine Lokalelementelektrolyse eintreten: An den Flächen negativeren Potentials geht Metall in Lösung, während die Flächen mit edlerem Potential als Elektronengeber arbeiten und durch Metallabscheidung wachsen. Dieser Prozeß läuft so lange, bis die an den einzelnen Einkristallbereichen sich nach rückwärts auflösenden Flächen negativsten Potentials alle übrigen Flächen „aufgefressen“ haben. Parallel damit geht eine Einstellung des beobachtbaren Gesamtpotentials auf einen negativen Endwert vor sich, indem zunächst wegen der Polarisation durch die Lokalströme ein positiveres Zwischenpotential auftritt.

Auch beim Auflösen von Metall in Säure, insbesondere beim Ätzen, ist der elektrolytische Gleichgewichtsmechanismus maßgebend, da diese Art von Korrosion durch elektrochemische Prozesse bestimmt wird. Auch hier sollte also nur die Flächenart mit dem unedelsten Potential erscheinen.

\section{Absolutes und relatives Potential}

Dadurch, daß das Metallelektrodenpotential nicht durch die Elektronenaustrittsarbeit am polykristallinen Metall $\psi_{\mathrm{P}}$, sondern - nach erfolgter Potentialeinstellung - durch die größte Halbkristall-Lagen-Aus-

42 T. E rdey-Gruz, Z. physik. Chem. Abt. A 172, 157 [1935].

43 I. N. S transk i, Z. physik. Chem. Abt. B 11, 342 [1930].

$44 \mathrm{Da}$ verschiedene Flächenarten das gleiche Ruhepotential haben, wollen wir jetzt als unwahrscheinlich betrachten.

45 Vgl. S. M a ki s hi m a 9 , S. 710. trittsarbeit $\psi_{\mathrm{H}}$ bestimmt ist, erfahren die theoretischen absoluten Potentialwerte eine Änderung, über deren Betrag sich vorerst allerdings noch nichts Genaues sagen läßt. In der Frage des absoluten Normalpotentials bestand nun bisher, selbst unter Berücksichtigung der beträchtlichen Unsicherheit der Meßergebnisse, eine erhebliche Diskrepanz zwischen Theorie und Experiment ${ }^{45}$, und zwar liegen die nach verschiedenen Methoden ${ }^{46}$ bestimmten absoluten Potentiale stets negativer als die nach Gl. (2) mit $\psi_{\mathrm{P}}$ berechneten. Diese Differenz kann durch eine erhöhte Elektronenaustrittsarbeit infolge Adsorption von $\mathrm{H}_{2} \mathrm{O}^{47}$ nicht erklärt werden, wie wir gezeigt haben, sondern ist sicher auf den Unterschied zwischen $\psi_{\mathrm{P}}$ und $\psi_{\mathrm{H}}$ zurückzuführen. Die Elektronenemission am polykristallinen Metall findet ja vorzugsweise an den Flächen kleinster Austrittsarbeit statt, so daß es durchaus möglich ist, daß die größte Halbkristall-Lagen-Austrittsarbeit $\psi_{\mathrm{H}}$, die wahrscheinlich kleiner ist als die größte Flächenaustrittsarbeit, den Wert $\psi_{\mathrm{P}}$ trotzdem noch wesentlich übersteigt.

Größere Bedeutung als das absolute Potential besitzt das auf eine Normalelektrode bezogene (relative) Potential, dieses ergibt sich, sofern Diffusionspotentiale im Elektrolyten vermieden werden, zu $E-E^{N}+K$, wo $E^{N}$ das absolute Potential der Normalelektrode und $K$ das Kontaktpotential zwischen den zwei Elektrodenmetallen ist. Da man $K$ als eine nur von der Art des Metalles abhängige Größe zu betrachten hat ( $K$ ist nach der Elektronentheorie gleich der Differenz der Fermi-Grenzenergien der zwei sich berührenden Metalle), so sollten alle Schlüsse bezüglich der Strukturabhängigkeiten des absoluten Potentials auch für das relative Potential Geltung haben. Bisher ${ }^{48}$ hatte man anscheinend für $K$ statt des inneren Kontaktpotentials (Galvanipotentials) das äußere Kontaktpotential (Voltapotential), welches, gleich der Differenz der Elektronenaustrittsarbeiten $\psi$ ist ${ }^{49}$, benutzt, so daß das relative Potential von $\psi$ unabhängig wurde. Die Wahl des äußeren Kontaktpotentials ${ }^{50}$ ist

46 Vgl. K. B ennewitz u. J. S chulz, Z. physik. Chem. Abt. A 124, 115 [1926]; J. B i ll i t e r, Z. Elektrochem. angew. physik. Chem. 37, 736 [1931].

47 A. E u c k e n, Lehrb. d. Chem. Physik II, 2 [1944], S. 1324.

48 S. M a kishima ${ }^{9}, A . E$ e k e n ${ }^{47}$

49 W. S ch ot tk y u. H. R o the, Hdb. Exp. Physik 12, 2 [1928], S. 145.

50 $\mathrm{Da}$ das äußere gleich dem inneren Kontaktpotential und die Austrittsarbeit gleich der Fermi-Grenzenergie ist, wie es z. B. bei H. Fröhlich, Elektronentheorie der Metalle, Berlin 1936; Physik. Z. Sowjetunion 7, 509 [1935], unter gewissen Voraussetzungen abgeleitet wird, läßt sich heute nicht mehr aufrechterhalten. 
jedoch im vorliegenden Fall nicht zulässig. An einer Flächenabhängigkeit auch des relativen Potentials ist also vorläufig festzuhalten.

\section{Mögliche Einschränkungen der Theorie}

Es ist wohl überflüssig, besonders darauf hinzuweisen, daß die obigen Betrachtungen in mancher Hinsicht etwas idealisierte Verhältnisse voraussetzen. $\mathrm{Ob}$ sich die Theorie in einem konkreten Einzelfall anwenden läßt, kann man bei der mẹist unzureichenden Kenntnis der tatsächlichen Oberflächenverhältnisse zunächst nur rückwärts feststellen. Immerhin aber dürften oben wohl die wesentlichsten Punkte besprochen worden sein. In diesem Kapitel wollen wir nur noch zweierlei erwähnen, was die obigen Aussagen der Theorie in Frage stellen könnte ${ }^{51}$ :

1. Es ist festgestellt worden, daß manche Flächenarten von Metallkristallen durch die eigene Schmelze nicht vollständig benetzbar sind ${ }^{52}$. Es wäre denkbar, daß etwas Ähnliches auch bei Berührung mit Elektrolyten der Fall ist. Die Aussagen über die Flächenabhängigkeit des Potentials wären dann zu modifizieren.

2. Gasbeladungen der Metalloberflächenschicht können den Elektrodenprozeß unmittelbar oder mittelbar beeinflussen und damit die Verhältnisse in schwer zu übersehender Weise ändern.

\section{Deutung einiger Experimente}

Zum Schluß wollen wir einige Versuchsergebnisse besprechen, die in den Zuständigkeitsbereich der oben entwickelten Theorie fallen und durch sie z. Tl. eine einfache Erklärung finden.

1. Die geforderte Potentialänderung an sehr feinkristallinen Metallen wurde in der Größe von etwa $30 \mathrm{mV}$ beobachtet und eine starke Neigung zur Rebzw. Sammelkristallisation, d.h. zum Wachsen der größeren Teilchen mit positiverem Potential, festgestellt ${ }^{53}$.

2. Ein eindeutiger Nachweis der Flächenabhängigkeit des Potentials durch Potentialmessung an isolierten Einkristallflächen liegt bisher nicht vor. In

51 Einige noch offene Probleme der Theorie der Elektronenemission lassen wir hierbei außer Betracht.

$52 \mathrm{M}$. V o lm e r u. O. S c h midt, Z. physik. Chem. Abt. B 35, 467 [1937]; I. N. S t r a n s k i, Naturwiss. 30, 425 [1942].

53 Kolloid-Z. 96, 226 [1941], Diskussionsbemerkungen $\mathrm{Hüt}$ ig u. Fricke.

54 P. A. A n d e r s o n, Nature [London] 123, 49 [1929]. älteren Arbeiten wurde lediglich folgendes gefunden: Nach Anders o ${ }^{54}$ ist bei $\mathrm{Zn}$ das Potential an (0001) identisch mit dem an polykristalliner Oberfäche, während mit zunehmender Flächenneigung gegen (0001) das Potential positiver zu werden scheint (beides wäre im Einklang mit obiger Theorie). Der letzte Punkt wird jedoch von $\mathrm{Str}$ a u m a n i s ${ }^{55}$ bestritten, allerdings wird hier zugleich bemerkt, daß die Kristallauflösung nicht in einer Parallelverschiebung der Flächen besteht. Die makroskopischen Flächen enthielten also mehrere Kristallfächenarten, so daß man aus den Messungen nichts Endgültiges entnehmen $\mathrm{kann}^{56}$.

3. Die unterschiedlichen Eigenschaften mechanisch erzeugter oder durch Ätzen elektrochemisch vorbehandelter Metalloberflächen sind durch Versuche von $\mathrm{Erb}$ a c h e $\mathrm{r}^{57}$ nachgewiesen worden. Erbacher stellte mit Hilfe radioaktiver Isotope an $\mathrm{Pb}$ und $\mathrm{Bi}$ fest, daß beim Einbringen der Metalle in ihre Salzlösungen ein elektrochemischer Austausch der Atome stattfindet, und zwar bei „mechanischer" Oberfläche in einer durchschnittlichen Schichtdicke von 100 Atomlagen, bei geätzter Oberfläche dagegen nur in einer Schichtdicke von höchstens 3 bis 5 Atomlagen. Dieses von Erbacher nicht überzeugend gedeutete Resultat läßt sich durch die im Kap. IV entwickelte Vorstellung einfach erklären. Übrigens dürfte sich die von Erbacher benutzte Indikatormethode leicht auch auf größere Einkristalle anwenden lassen, bei denen Lokalanoden und -kathoden so weit auseinander liegen, daß sie radiographisch getrennt festgestellt werden können; dieses würde definiertere Aussagen ermöglichen.

4. Bei $\mathrm{Al}$ wurden von $\mathrm{Mahl}$ und $\mathrm{Stranski}{ }^{58}$ Ätzfiguren elektronenmikroskopisch untersucht, wobei allein Flächen (001) zu beobachten waren. Dieses Ergebnis läßt sich durch die Annahme erklären, daß (001) die größte Elektronenaustrittsarbeit und damit das negativste Potential hat, was im Einklang mit den Verhältnissen bei $\mathrm{Cu}$ und $\mathrm{Ag}$ steht (Kap. II). Nach dem Stranskischen Gleichgewichtsmechanismus sollten dagegen in größter Ausdehnung die Flächen (111) und dazu weitere Flächenarten erscheinen. Stranski erklärte die Abweichung dadurch, daß die gefundene

55 M. Str a u ma nis, Z. physik. Chem. Abt. A 147, 161 [1930].

56 Im Anschluß an Straumanis entwickelte M. S a to, Sci. Rep. Tôhoku Imp. Univ., Ser. I 25, 829 [1936], eine Theorie, die aber auf sehr unsicheren Voraussetzungen basiert.

57 O. E rba cher, Korros. u. Metallschutz 17, 5 [1941]; Z. Elektrochem. 53, 51, 67 [1949], Versuchsgruppe 3 u. 10.

$58 \mathrm{H}$. M a h l u. I. N. Stranski, Z. physik. Chem. Abt. B 51, 319 [1942]. 
Gleichgewichtsform die Lösungsform eines zweidimensionalen Oberflächenoxyds sein soll, was aber mit anderen Beobachtungen nicht vereinbar ist ${ }^{59}$. Bei der Ätzung mit trockenem $\mathrm{HCl}^{\mathrm{c} 0}$ verläuft die Korrosion nicht elektrochemisch, so daß wir hierauf nicht weiter einzugehen brauchen.

Die elektrolytischen Kristallwachstumsversuche an $\mathrm{Ag}$ und $\mathrm{Cu}$, die Erdey-Gruz ${ }^{61}$ zur Prüfung der

59 M. S t r a u m a n i s, Korros. u. Metallschutz 19, 157 [1943].

co H. Mahl u. I. N. Stranski, Z. physik. Chem. Abt. B 52, 257 [1942].

61 T. Erdey-Gruz, Z. physik. Chem. Abt. A 172, 157 [1935]; Abt. A 178, 266 [1937].
Stranskischen Theorie anstellte, führten meist zu zwei oder drei verschiedenen Flächenarten. Vielleicht ist aber die beobachtete Endform keine Gleichgewichtsform, da in komplexen Lösungen, die Erdey-Gruz ausschließlich benutzte, die Abscheidungspolarisation schon bei kleineren Stromdichten beträchtliche Werte annimmt und dadurch die Stromverteilung an den einzelnen Flächenarten völlig geändert werden kann. Schon aus diesem Grunde brauchen die hier gefundenen Ergebnisse nicht im Widerspruch zu unserer Theorie zu stehen.

Hrn. Prof. Dr. H. S t a u de danke ich für sein Interesse und mehrere Diskussionen.

\section{NOTIZEN}

\section{Großkreisausbrechungen bei Kurzwellen}

Von Walter Budde*

(Z. Naturforschg. 5 a, 291 [1550]; eingeg. am 25. März 1950)

In Koelby (Dänemark) wurde die Strahlung eines in München befindlichen Senders empfangen und die Abweichungen der Wellen vom Großkreis München-Koelby gemessen. Als richtungsempfindliches System wurde eine Zweibasisanlage ${ }^{1}$ verwendet. Infolge Zugrundelegung des Großbasisprinzips von v. $\mathrm{Handel} 2$ wurden die durch Rückstrahler und Mehrwegeausbreitung der Wellen entstehenden Fehler klein und es ließen sich Messungen von Großkreisausbrechungen mit großer Genauigkeit durchführen.

Die Meßergebnisse zeigen einen tageszeitlichen Gang der Großkreisausbrechungen, der sich durch den tageszeitlichen Gang der Schräglage der Ionosphäre (morgens im Osten, abends im Westen tiefer) einwandfrei erklären läßt. Weiterhin treten kurzzeitige Schwankungen der Empfangsrichtung auf. (Größenordnung der Schwankungsdauer: einige Minuten.) Zu Zeiten brauchbaren Empfanges lag die Empfangsrichtung in $70 \%$ der Zeit innerhalb von $\pm 1,5^{\circ}$, zu $100 \%$ innerhalb von $\pm 2,5^{\circ}$ um den Mittelwert der Empfangsrichtung. (Mittelwerte über einige Monate.)

* Aachen, Karl-Marx-Allee 154.

1 W. B u d d e, Diss. T. H. München 1947.

2 P. v. $\mathrm{H}$ andel, Interferenz-Hyperbeln, DVL-Bericht 1934. P. v. H and el, Großbasisverfahren Komet, DVL-Bericht 1942.
Grcßkreisausbrechungen größer als $\pm 2,5^{\circ}$ wurden nicht festgestellt. Die Sendefrequenz betrug $9830 \mathrm{kHz}$.

Die Versuche wurden durchgeführt in den Jahren 1943 bis 1945 an dem unter Leitung von Hrn. Prof. v. H a n del stchenden Ferdinand-Braun-Institut in Landsberg a. Lech. Eine ausführliche Mitteilung erfolgt im Archiv der elektrischen Übertragung.

\section{Die Bedeutung des Stickoxydes für die Trägerdichte in einer Niederstrombogensäule in Luft \\ ( $\mathrm{N}$ a c h t r a g) \\ Von K.-H. H ö c k e r* \\ (Z. Naiurforschg. 5 a, 291 [1950]; eingeg. am 15. April 1950)}

In meiner Arbeit unter obigem Titel ${ }^{1}$ wurde der Anteil des Stickoxydes an der Trägerdichte im Lichtbogen eingehend quantitativ behandelt. Auf Wunsch von Hrn. v. Engel will ich gern nachtragen, daß v. Engel und $\mathrm{S}$ t e e $\mathrm{nbeck}$ auf die Beteiligung des NO hingewiesen haben 2. Durch die Schwierigkeiten in der Literaturbeschaffung und sonstiger Behinderungen nach dem Kriege war es mir derzeit nicht möglich, dem Ursprung der Diskussion über den NO-Anteil nachzugehen.

* Stuttgart-Zuffenhausen, Zabergäustr. 62.

1 K.-H. H ö c k e r, Z. Naturforschg. 1, 384 [1946].

2 A. v. Engel u. M. S t e e $\mathrm{n} b$ e ck, Wiss. Veröff. Siemens-Werken 12, 74 [1932], Elektrische Gasentladungen, Berlin 1934, S. 144.

\section{BESPRECHUNGEN}

Lehrbuch der Theoretischen Physik. Von Walter We i zel. Bd. I. Physik der Vorgänge. Bewegung, Elektrizität, Licht, Wärme. Springer-Verlag, Berlin-Göttingen-Heidelberg 1949. XIII/771 S. mit 270 Abb., Preis geb. DM 56.90.

Den Verf. hat zur Herausgabe eines neuen Lehrbuchs der Theoretischen Physik vor allem die Aufgabe verlockt, den Problemkreis der Quantentheorie organisch in die Darstellung einzufügen, wobei er allerdings gleich einen Vorbehalt macht in bezug auf die Theorie der Atomkerne, die er vorläufig noch als „etwas kümmerliches Anhängsel“ behandelt. Wieweit der Versuch eines einheitlichen Aufbaus der Theoretischen Physik gelungen ist, wird erst der zweite Band zeigen können, da in ihm ge- 